\title{
Preface to special issue on Advances in Cloud Computing
}

\author{
Chunming Rong • Frode Eika Sandnes • \\ Rajkumar Buyya
}

Published online: 28 April 2012

(C) Springer Science+Business Media, LLC 2012

In 2008 the two first guest editors of this special issue, Chunming Rong and Frode Eika Sandnes, drafted the ideas for the first international conference on cloud computing during a conference in Hainan Island, China. One year later the conference became a reality and the response and interest was overwhelming. Rong and Sandnes later invited the third guest editor, Rajkumar Buyya, to join the guest editorial team.

This special issue entitled Advances in Cloud Computing presents the best papers presented at the first international conference on Cloud Computing (CloudCom) that was held December 1-4 in Beijing China. It has grown to be a premiere venue on cloud computing research, and has since been hosted in Indianapolis, USA (2010), Athens, Greece (2011) and in 2012 CloudCom will be hosted in Taipei, Taiwan.

Of the more than 100 papers finally accepted for presentation at CloudCom 2009 the ones that received the best review reports were invited to submit revised and extended versions of their manuscripts and these underwent several rounds of review. Of the invited papers only the handful presented herein were accepted for inclusion in this special issue.

\footnotetext{
C. Rong

University of Stavanger, Stavanger, Norway

e-mail: chunming.rong@uis.no
}

F.E. Sandnes $(\varangle)$

Faculty of Technology, Art and Design, Oslo and Akershus University College of Applied Sciences, Oslo, Norway

e-mail: frode-eika.sandnes@hioa.no

R. Buyya

Department of Computing and Information Systems, The University of Melbourne, Melbourne, Australia

e-mail: raj@csse.unimelb.edu.au 
Thematically, the papers showcase the diversity of research that is currently conducted within the domain of cloud computing, that is, administration, security, performance, analysis of social structures in clouds, and resource management.

In his paper "Simplified cloud-oriented virtual machine management with MLN", Begnum demonstrates how his tool MLM reduces the complexity of virtual machine management applied to clouds from the users' perspective. With MLM users do not need specialist knowledge about virtual machines-it is all embedded in the tool.

Mowbray, Pearson and Shen describe a privacy manager for cloud computing in their paper "Enhancing privacy in cloud computing via policy-based obfuscation". This policy-based management helps users protect their private data within the cloud service and help providers conform to privacy laws. The authors also address the scalability of the approach and performance issues.

In the contribution "Coordinated load management in Peer-to-Peer coupled federated grid systems" Ranjan, Harwood, and Buyya propose the idea of a coordinated load management protocol for P2P grids managed using a distributed hash table. The coordination protocol has a message complexity that is logarithmic to the number of nodes in the system.

Liu, Zhou, Wang, Zhang and Liu take a different look at clouds in their paper entitled "Irregular community discovery for cloud service improvement", where a new strategy for discovering irregular communities and social structures within clouds is investigated. These irregular patterns can among other things be used to identify prominent customers.

Next, Zhao, Rong, Jaatun and Sandnes explore five reference deployment models in their paper "Reference deployment models for eliminating user concerns on cloud security". The five models provide different security features that suit different requirements and scenarios.

Finally, in the paper entitled "Composable IO: a novel resource sharing platform in personal Clouds" Wu, Wang, Lin, and Miao address the interesting issue of sharing IO peripherals among Cloud users, in particular USB-devices. The approach is based on pro-migration USB access where a migrated application running on the target host can access the USB peripheral on the source host as a supplement to traditional virtual machine migration.

Finally, we want to thank the Editor-in-Chief Hamid R. Arabnia for giving us the opportunity to realize this special issue, the anonymous reviewers for their patience and hard work reviewing the manuscripts and suggesting improvements, and the editorial staff for their excellent service.

Stavanger, Oslo, Melbourne

April 20, 2012 